\title{
Mu-tau neutrino refraction and collective three-flavor transformations in supernovae
}

\author{
Andreu Esteban-Pretel, ${ }^{1}$ Sergio Pastor, ${ }^{1}$ Ricard Tomàs, ${ }^{1}$ Georg G. Raffelt,${ }^{2}$ and Günter Sigl ${ }^{3,4}$ \\ ${ }^{1}$ Institut de Física Corpuscular, CSIC-Universitat de València, Ed. Instituts d'Investigació, Ap. correus 22085, 46071 València, Spain \\ ${ }^{2}$ Max-Planck-Institut für Physik, Werner-Heisenberg-Institut, Föhringer Ring 6, 80805 München, Germany \\ ${ }^{3}$ II. Institut für theoretische Physik, Universität Hamburg, Luruper Chaussee 149, 22761 Hamburg, Germany \\ ${ }^{4} A P C^{*}$ (AstroParticules et Cosmologie), 10, rue Alice Domon et Léonie Duquet, 75205 Paris Cedex 13, France
}

(Received 17 December 2007; published 27 March 2008)

We study three-flavor collective neutrino transformations in the dense-neutrino region above the neutrino sphere of a supernova core. We find that two-flavor conversions driven by the atmospheric mass difference and the 13-mixing angle capture the full effect if one neglects the second-order difference between the $\nu_{\mu}$ and $\nu_{\tau}$ refractive index. Including this "mu-tau matter term" provides a resonance at a density of $\rho \approx 3 \times 10^{7} \mathrm{~g} \mathrm{~cm}^{-3}$ that typically causes significant modifications of the overall $\nu_{e}$ and $\bar{\nu}_{e}$ survival probabilities. This effect is surprisingly sensitive to deviations from maximal 23-mixing, being different for each octant.

DOI: 10.1103/PhysRevD.77.065024

PACS numbers: $14.60 . P q, 97.60 . \mathrm{Bw}$

\section{INTRODUCTION}

Neutrinos of different flavor suffer different refraction in matter [1]. The energy shift between $\nu_{e}$ and $\nu_{\mu}$ or $\nu_{\tau}$ is $\Delta V=\sqrt{2} G_{\mathrm{F}} Y_{e} n_{B}$ with $G_{\mathrm{F}}$ the Fermi constant, $n_{B}$ the baryon density, and $Y_{e}=n_{e} / n_{B}$ the electron fraction. $\Delta V$ is caused by the charged-current $\nu_{e}$-electron interaction that is absent for $\nu_{\mu}$ and $\nu_{\tau}$. For a matter density $\rho=$ $1 \mathrm{~g} \mathrm{~cm}^{-3}$ we have $\sqrt{2} G_{\mathrm{F}} n_{B}=7.6 \times 10^{-14} \mathrm{eV}$, yet this small energy shift is large enough to be of almost universal importance for neutrino oscillation physics.

In normal matter, $\mu$ and $\tau$ leptons appear only as virtual states in radiative corrections to neutral-current $\nu_{\mu}$ and $\nu_{\tau}$ scattering, causing a shift $\Delta V_{\mu \tau}=\sqrt{2} G_{\mathrm{F}} Y_{\tau}^{\text {eff }} n_{B}$ between $\nu_{\mu}$ and $\nu_{\tau}$. It has the same effect on neutrino dispersion as real $\tau$ leptons with an abundance [2]

$$
Y_{\tau}^{\text {eff }}=\frac{3 \sqrt{2} G_{\mathrm{F}} m_{\tau}^{2}}{(2 \pi)^{2}}\left[\ln \left(\frac{m_{W}^{2}}{m_{\tau}^{2}}\right)-1+\frac{Y_{n}}{3}\right]=2.7 \times 10^{-5},
$$

where $n_{e}=n_{p}$ was assumed. For the neutron abundance we have used $Y_{n}=n_{n} / n_{B}=0.5$, but it provides only a $2.5 \%$ correction so that its exact value is irrelevant. A large nonstandard contribution to $Y_{\tau}^{\text {eff }}$ can arise from radiative corrections in supersymmetric models [3], but we will here focus on the standard model effect alone.

This "mu-tau matter effect" modifies oscillations if $\Delta V_{\mu \tau} \gtrsim \Delta m^{2} / 2 E$. For propagation through the Earth and for $\Delta m_{\mathrm{atm}}^{2}=2-3 \times 10^{-3} \mathrm{eV}^{2}$, this occurs for neutrino energies $E \gtrsim 100 \mathrm{TeV}$. The oscillation length then far exceeds $r_{\text {Earth }}$ so that $\Delta V_{\mu \tau}$ is irrelevant for the high-energy neutrinos that are searched for by neutrino telescopes.

Alternatively, the mu-tau matter effect can be important at the large densities encountered by neutrinos streaming

\footnotetext{
*UMR 7164 (CNRS, Université Paris 7, CEA, Observatoire de Paris)
}

off a supernova (SN) core [4]. For $E=20 \mathrm{MeV}$ the condition $\Delta V_{\mu \tau}=\Delta m_{\mathrm{atm}}^{2} / 2 E$ implies $\rho \approx 3 \times 10^{7} \mathrm{~g} \mathrm{~cm}^{-3}$. Numerical SN density profiles [5] reveal that this occurs far beyond the shock-wave radius during the accretion phase, but retracts close to the neutrino sphere after the explosion has begun. To illustrate this point we show in Fig. 1 the same matter density profiles as in Ref. [5] at $1 \mathrm{~ms}$

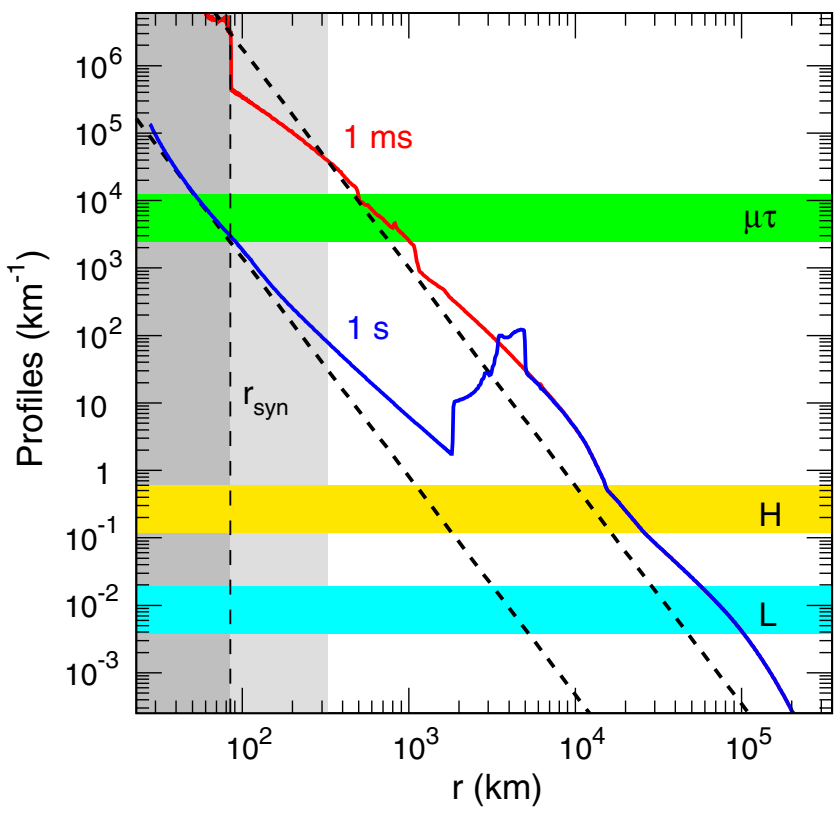

FIG. 1 (color online). Density profiles in terms of the weak potential $\Delta V=\sqrt{2} G_{\mathrm{F}} n_{e}$ at $1 \mathrm{~ms}$ and $1 \mathrm{~s}$ post bounce of the numerical SN models described in Ref. [5] (solid lines). The dashed lines represent the simplified matter profile of Eq. (4) for $\lambda_{0}=4 \times 10^{6} \mathrm{~km}^{-1}$ and $\lambda_{0}=5 \times 10^{9} \mathrm{~km}^{-1}$, used in our numerical calculations in Fig. 2. As horizontal bands we indicate the conditions $\Delta V_{\mu \tau}=\Delta m_{\mathrm{atm}}^{2} / 2 E, \Delta V=\Delta m_{\mathrm{atm}}^{2} / 2 E$, and $\Delta V=\Delta m_{\mathrm{sol}}^{2} / 2 E$ for a typical range of $\mathrm{SN}$ neutrino energies. The gray shaded range of radii corresponds to the region of collective neutrino transformations. Within the radius $r_{\text {syn }}$ the collective oscillations are of the synchronized type. 
post bounce (lighter, red line) and at $1 \mathrm{~s}$ post bounce (darker, blue line). As a green (upper) horizontal band we indicate the condition $\Delta V_{\mu \tau}=\Delta m_{\mathrm{atm}}^{2} / 2 E$ for a typical range of $\mathrm{SN}$ neutrino energies, whereas the yellow (middle) and light-blue (lower) bands indicate the densities corresponding to the H-resonance (driven by $\Delta m_{\mathrm{atm}}^{2}$ ) and the L-resonance (driven by $\Delta m_{\text {sol }}^{2}$ ). The $\nu_{\mu}, \nu_{\tau}, \bar{\nu}_{\mu}$, and $\bar{\nu}_{\tau}$ fluxes from a $\mathrm{SN}$ are virtually identical, leaving the $\mu \tau$-resonance moot, whereas the $\mathrm{H}$ - and L-resonances cause well-understood consequences that are completely described by the energy-dependent swapping probabilities for $\nu_{e}$ and $\bar{\nu}_{e}$ with some combination $\nu_{x}$ of the $\mu$ and $\tau$ flavor [6]. Therefore, the traditional view has been that genuine three-flavor effects play no role for SN neutrino oscillations unless mu and tau neutrinos are produced with different fluxes [4].

In a recent series of papers [7-22] it was recognized, however, that the traditional picture was not complete: neutrino-neutrino interactions cause large collective flavor transformations in the SN region out to a few $100 \mathrm{~km}$ (gray shaded region in Fig. 1). With the exception of Refs. [21,22], only two-flavor conversions driven by $\Delta m_{\text {atm }}^{2}$ and the small $\Theta_{13}$ have thus far been studied.

We here extend our previous numerical solutions [17] to the case of three neutrino flavors. Our main results can be summarized as follows: (i) A two-flavor treatment indeed captures the full effect if one ignores $\Delta V_{\mu \tau}$ and if the ordinary Mikheyev-Smirnov-Wolfenstein (MSW) resonances occur outside of the collective neutrino region. (ii) Including $\Delta V_{\mu \tau}$ strongly modifies the $\nu_{e}$ or $\bar{\nu}_{e}$ survival probabilities, influencing the neutrino signal from the next galactic SN. (iii) The effect depends sensitively on a possible deviation from maximal $\Theta_{23}$. The purpose of our paper is to provide a first illustration of these findings that no doubt need to be refined in the future.

Our work is organized as follows. In Sec. II we present the equations of motion which are solved for the threeflavor neutrino fluxes in a simplified scenario for the $\mathrm{SN}$ environment. In Sec. III we consider the limit of a vanishing $\mu \tau$ matter effect, while our results when it is significant are described in Sec. IV. We conclude in Sec. V.

\section{EQUATIONS OF MOTION}

Mixed neutrinos are described by matrices of density $\rho_{\mathbf{p}}$ and $\bar{\rho}_{\mathbf{p}}$ for each (anti)neutrino mode. The diagonal entries are the usual occupation numbers whereas the off-diagonal terms encode phase information. The equations of motion (EOMs) are

$$
\text { i } \partial_{t} \varrho_{\mathbf{p}}=\left[\mathrm{H}_{\mathbf{p}}, \varrho_{\mathbf{p}}\right]
$$

where the Hamiltonian is [23]

$$
\mathrm{H}_{\mathbf{p}}=\Omega_{\mathbf{p}}+\mathrm{V}+\sqrt{2} G_{\mathrm{F}} \int \frac{\mathrm{d}^{3} \mathbf{q}}{(2 \pi)^{3}}\left(\varrho_{\mathbf{q}}-\bar{\varrho}_{\mathbf{q}}\right)\left(1-\mathbf{v}_{\mathbf{q}} \cdot \mathbf{v}_{\mathbf{p}}\right),
$$

$\mathbf{v}_{\mathbf{p}}$ being the velocity. The matrix of vacuum oscillation frequencies is $\Omega_{\mathbf{p}}=\operatorname{diag}\left(m_{1}^{2}, m_{2}^{2}, m_{3}^{2}\right) / 2|\mathbf{p}|$ in the mass basis. The matter effect is represented, in the weakinteraction basis, by $\mathrm{V}=\sqrt{2} G_{\mathrm{F}} n_{B} \operatorname{diag}\left(Y_{e}, 0, Y_{\tau}^{\text {eff }}\right)$. For antineutrinos the only difference is $\Omega_{\mathbf{p}} \rightarrow-\Omega_{\mathbf{p}}$.

In spherical symmetry the EOMs can be expressed as a closed set of differential equations along the radial direction $[17,20]$. We solve them numerically as previously described [17], now using $3 \times 3$ matrices instead of polarization vectors. The factor $\left(1-\mathbf{v}_{\mathbf{q}} \cdot \mathbf{v}_{\mathbf{p}}\right)$ in the Hamiltonian implies "multiangle effects" for neutrinos moving on different trajectories [8,9,11]. However, for realistic SN conditions the modifications are small, allowing for a single-angle approximation. We implement this approximation by launching all neutrinos with $45^{\circ}$ relative to the radial direction [17].

As a further simplification we use a monochromatic spectrum $(E=20 \mathrm{MeV})$, ignoring the "spectral splits" caused by collective oscillation effects $[11,15,16,18,20]$. Oscillation effects require flavor-dependent flux differences. One expects $F_{\nu_{e}}>F_{\bar{\nu}_{e}}>F_{\nu_{\mu}}=F_{\bar{\nu}_{\mu}}=F_{\nu_{\tau}}=$ $F_{\bar{\nu}_{\tau}}$. The equal parts of the fluxes drop out of the EOMs, so as the initial condition we use $F_{\nu_{\mu}, \bar{\nu}_{\mu}, \nu_{\tau}, \bar{\nu}_{\tau}}=0$ and $F_{\nu_{e}}=(1+\epsilon) F_{\bar{\nu}_{e}}$ with $\epsilon=0.25$.

For the neutrino parameters we use $\Delta m_{12}^{2}=\Delta m_{\text {sol }}^{2}=$ $7.6 \times 10^{-5} \mathrm{eV}^{2}, \quad \Delta m_{13}^{2}=\Delta m_{\mathrm{atm}}^{2}=2.4 \times 10^{-3} \mathrm{eV}^{2}$, $\sin ^{2} \Theta_{12}=0.32, \sin ^{2} \Theta_{13}=0.01$, and a vanishing Dirac phase $\delta=0$, all consistent with measurements [24-26]. We consider the entire allowed range $0.35 \leq \sin ^{2} \Theta_{23} \leq$ 0.65 because our results depend sensitively on $\Theta_{23}$.

We use a fixed matter profile of the form $\rho \propto r^{-3}$, implying a radial variation of the weak potential of

$$
\Delta V=Y_{e} \lambda_{0}\left(\frac{R}{r}\right)^{3}
$$

where $R=10 \mathrm{~km}$ is our nominal neutrino-sphere radius and $Y_{e}=0.5$. In Fig. 1 we show this profile (dashed lines) for two different values of $\lambda_{0}=4 \times 10^{6} \mathrm{~km}^{-1}$ and $\lambda_{0}=$ $5 \times 10^{9} \mathrm{~km}^{-1}$. For the former case, the H-resonance is at $r_{\mathrm{H}}=1.9 \times 10^{3} \mathrm{~km}$, the L-resonance at $r_{\mathrm{L}}=8.3 \times$ $10^{3} \mathrm{~km}$, and the $\mu \tau$-resonance at $r_{\mu \tau}=71 \mathrm{~km}$. For the latter they are at $r_{\mathrm{H}}=2.0 \times 10^{4} \mathrm{~km}, r_{\mathrm{L}}=9.0 \times 10^{4} \mathrm{~km}$, and $r_{\mu \tau}=760 \mathrm{~km}$. $^{1}$

The strength of the neutrino-neutrino interaction can be parametrized by

\footnotetext{
${ }^{1}$ We loosely refer to the radius where $\Delta m_{\mathrm{atm}}^{2} / 2 E=\Delta V_{\mu \tau}$ as the $\mu \tau$ resonance, although this would be correct only for a small vacuum mixing angle in the 23 -subsystem.
} 


$$
\mu_{0}=\sqrt{2} G_{\mathrm{F}}\left(F_{\bar{\nu}_{e}}^{R}-F_{\bar{\nu}_{x}}^{R}\right),
$$

where the fluxes are taken at the neutrino-sphere radius $R$. As in our previous work [17] we shall assume $\mu_{0}=7 \times$ $10^{5} \mathrm{~km}^{-1}$. In the single-angle approximation where all neutrinos are launched with $45^{\circ}$ relative to the radial direction [17], the radial dependence of the neutrinoneutrino interaction strength can be explicitly written as

$$
\mu(r)=\mu_{0} \frac{R^{4}}{r^{4}} \frac{1}{2-R^{2} / r^{2}} .
$$

While the $r^{-4}$ scaling of $\mu(r)$ for $r \gg R$ is generic, the overall strength $\mu_{0}$ depends on the neutrino fluxes and on their angular divergence, i.e., on the true radius of the neutrino sphere. Our $R=10 \mathrm{~km}$ is not meant to represent the physical neutrino sphere, it is only a nominal radius where we fix the inner boundary condition for our calculation.

The collective neutrino oscillations are of the synchronized type within the "synchronization radius." For our chosen $\mu_{0}$ and for the assumed excess $\nu_{e}$ flux of $25 \%$ we find $r_{\text {syn }} \simeq 100 \mathrm{~km}$ as indicated in Fig. 1. Collective flavor transformations occur at $r>r_{\mathrm{syn}}$. Therefore, the $\mu \tau$ matter effect can be important only if it is sufficiently large for $r>r_{\text {syn }}$.

Figure 1 illustrates that the region where the $\mu \tau$-resonance takes place depends on the time after bounce. For realistic values of the matter density profile and neutrino-neutrino interaction, one expects $r_{\mu \tau}$ to lie far beyond the collective region at early times. This can be inferred from the relative position of $r_{\mathrm{syn}}$ and the intersection of the $1 \mathrm{~ms}$ profile and the green (upper) band. At later times though the proto neutron star contracts and $r_{\mu \tau}$ moves to smaller radii. Eventually $r_{\mu \tau}$ becomes smaller than $r_{\text {syn }}$, at which point $\Delta V_{\mu \tau}$ becomes irrelevant.

In order to mimic these different situations we will us a simple power-law matter profile of the form in Eq. (4). In other words, we will use a mu-tau matter potential of the form

$$
\Delta V_{\mu \tau}=Y_{\tau}^{\mathrm{eff}} \lambda_{0}\left(\frac{R}{r}\right)^{3},
$$

with a fixed $Y_{\tau}^{\text {eff }}$ given by Eq. (1) and a variable coefficient $\lambda_{0}$. Therefore early and late times can be reproduced by considering large and small values of $\lambda_{0}$, respectively, as can be seen in Fig. 1. In other words, we will always assume that the ordinary MSW resonances are far outside of the collective neutrino region, whereas the $\mu \tau$ resonance can lie at smaller (vanishing $\mu \tau$ matter effect) or larger (large $\mu \tau$ matter effect) radii than $r_{\text {syn }}$.

\section{VANISHING MU-TAU MATTER EFFECT}

As a first case we consider the traditional assumption of a vanishing $\mu \tau$ matter effect, which we account for using a value of $\lambda_{0}=4 \times 10^{6}$. We assume an inverted $\Delta m_{\mathrm{atm}}^{2}$ and use a nonmaximal value $\sin ^{2} \Theta_{23}=0.4$. Our numerical calculations for this case are shown in the top row of Fig. 2. The first two panels correspond to the radial evolution of the fluxes of the weak-interaction eigenstates of neutrinos and antineutrinos, respectively, whereas in the last two panels we show the evolution of the propagation eigenstates. These are the eigenstates of $\Omega_{\mathbf{p}}+\mathrm{V}$, i.e., of that part of the Hamiltonian Eq. (3) that does not include the neutrino-neutrino interactions. In the collective neutrino region, we observe the usual pair conversion of the $\nu_{e}$ and $\bar{\nu}_{e}$ fluxes into the $\mu$ and $\tau$ flavors. Had we chosen a maximal 23-mixing angle, the appearance curves for these flavors would be identical.

For larger distances the evolution consists of ordinary MSW transformations that are best pictured in the basis of instantaneous propagation eigenstates in matter (last two panels). Beyond the collective transformation region, all neutrinos and antineutrinos stay fixed in their propagation eigenstates. In the weak-interaction basis, on the other hand, this implies fast oscillations because we have a fixed energy, preventing kinematical decoherence between different energy modes. In the panels for neutrino and antineutrino interaction states, for radii beyond the denseneutrino region we show as thick lines the average evolution as well as the envelopes of the fast-oscillating flavor fluxes.

Another way of describing this evolution is by the level crossing schemes of Fig. 3. The upper panel represents the case of vanishing $\Delta V_{\mu \tau}$, corresponding to Fig. $5 \mathrm{~d}$ of Ref. [6]. The central panel represents the case with large $\Delta V_{\mu \tau}$ and a 23-mixing angle in the first octant and is similar to Fig. 2 of Ref. [4]. In such plots one shows the neutrino energy levels as a function of the matter density. The continuation of this diagram to negative densities gives us the energy levels of antineutrinos: the neutrino energy at a negative density really means the antineutrino energy at the corresponding positive density. For vanishing density (vacuum), we have the three vacuum mass eigenstates that are identical for neutrinos and antineutrinos. The blue (upper) line corresponds to propagation eigenstate 2 , the green (middle) line to 1, and the red (bottom) line to 3, a scheme representing the inverted hierarchy case. These lines represent the propagation eigenstates that are adiabatically connected for different densities.

While in vacuum the propagation eigenstates coincide with the mass eigenstates, at large densities they correspond to weak-interaction eigenstates. For vanishing $\Delta V_{\mu \tau}$ and at the low energies relevant to our problem, the $\mu$ and $\tau$ flavor are not distinguishable so that any convenient linear combination can be chosen as interaction eigenstates. It is convenient to introduce the states $\nu_{\mu}^{\prime}$ and $\nu_{\tau}^{\prime}$ that correspond to a vanishing 23-mixing angle, i.e., they diagonalize the 23-subsystem. If the small 13-mixing angle were to vanish, the 3-mass eigenstate would coincide with $\nu_{\tau}^{\prime}$. In 

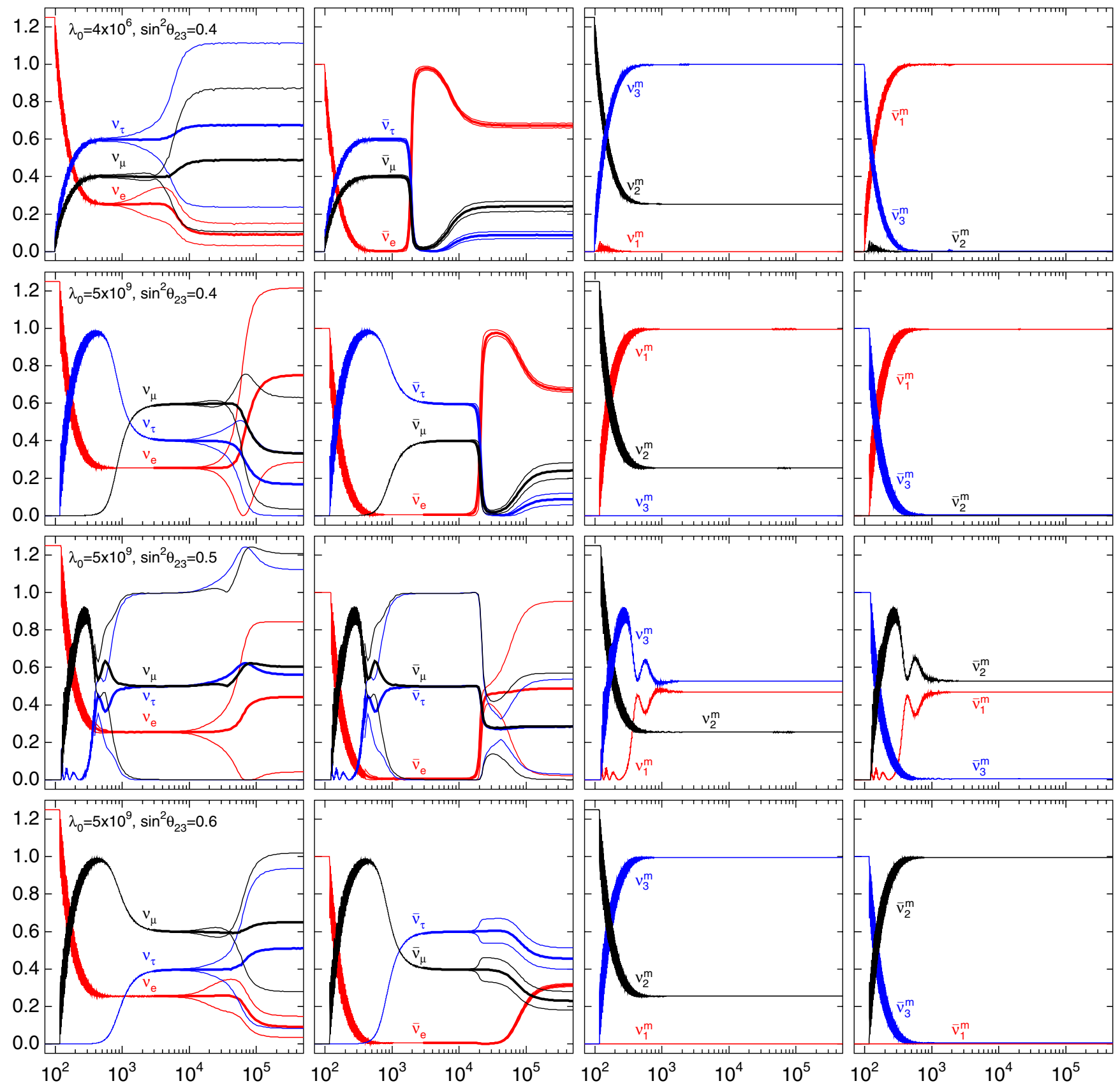

$r(\mathrm{~km})$

FIG. 2 (color online). Radial evolution of the neutrino fluxes, normalized to the initial $\bar{\nu}_{e}$ flux, for a fixed neutrino energy $\left(E_{\nu}=\right.$ $20 \mathrm{MeV}$ ) and an inverted $\Delta m_{\mathrm{atm}}^{2}$. From left to right: neutrino weak eigenstates, antineutrino weak eigenstates, neutrino propagation eigenstates, and antineutrino propagation eigenstates. In the first two columns, after bipolar conversions we show the average as thick lines and the envelopes of the fast-oscillating curves as thin lines. The top row shows the case of a vanishing $\mu \tau$ matter effect, while the three bottom rows use a large $\mu \tau$ effect with different values for the 23-mixing angle as indicated.

the upper panel of Fig. 3 and using the $\left(\nu_{e}, \nu_{\mu}^{\prime}, \nu_{\tau}^{\prime}\right)$ basis, the 2-state connects adiabatically to $\nu_{e}$ and $\bar{\nu}_{\mu}^{\prime}$, whereas the 3-state connects adiabatically to $\bar{\nu}_{e}$ and $\nu_{\tau}^{\prime}$.

At the neutrino sphere, the fluxes are prepared in $\nu_{e}$ and $\bar{\nu}_{e}$ eigenstates, which in the case of inverted mass hierarchy coincide with the propagation (or matter) eigenstates $\nu_{2}^{\mathrm{m}}$ and $\bar{\nu}_{3}^{\mathrm{m}}$, respectively. In the absence of neutrino-neutrino interactions, since the L-resonance is always adiabatic, the $\nu_{e}$ 's leave the star as $\nu_{2}$. In the case of $\bar{\nu}_{e}$ the evolution depends on $\sin ^{2} \Theta_{13}$ [6]. For values larger than $10^{-3}$ they propagate also adiabatically (MSW transformation) and escape as $\bar{\nu}_{3}$, whereas for values smaller than $10^{-5}$ the transition at the H-resonance is strongly nonadiabatic: there is a jump of matter eigenstates from $\bar{\nu}_{3}^{\mathrm{m}}$ to $\bar{\nu}_{1}^{\mathrm{m}}$ and 

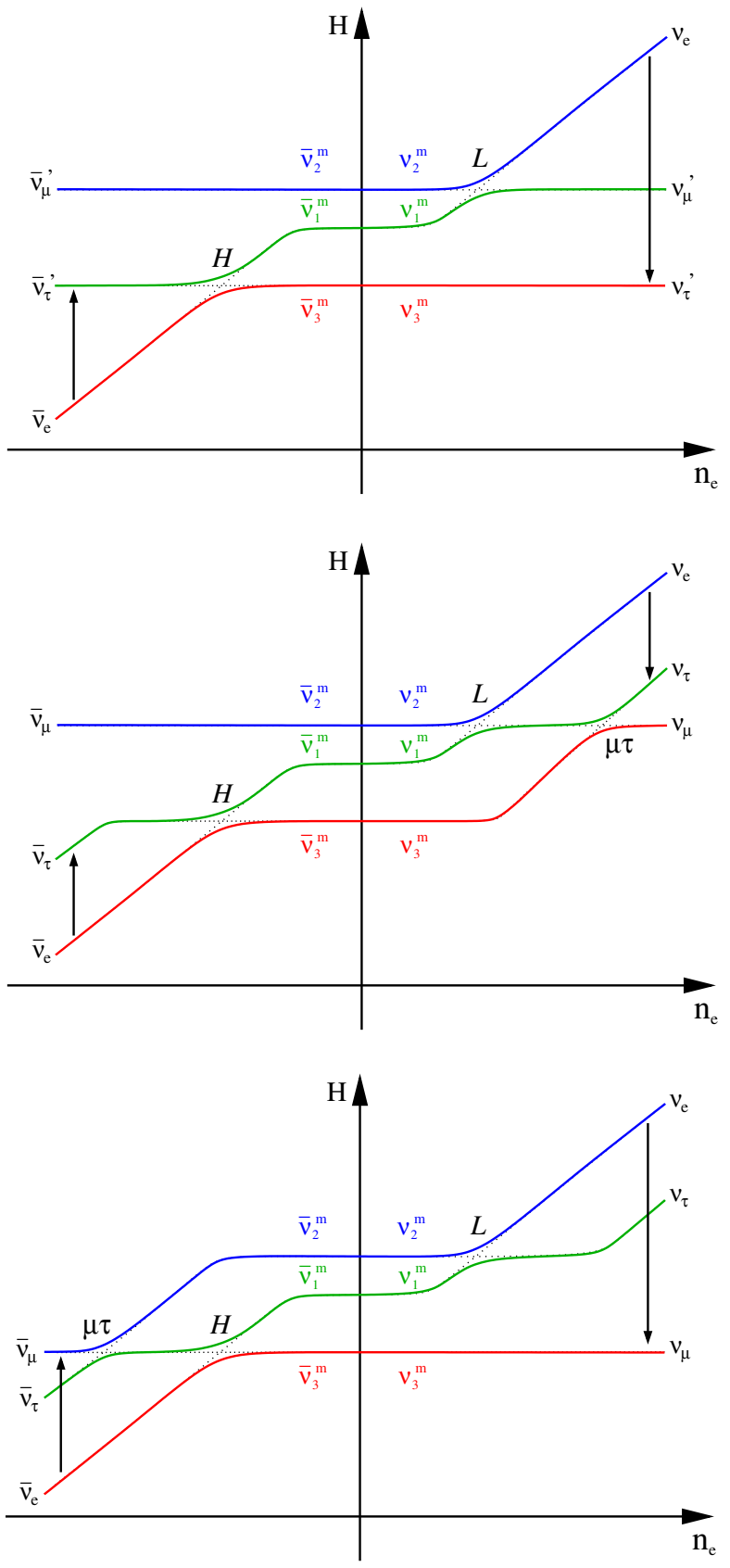

FIG. 3 (color online). Level crossing scheme of neutrino conversion for the inverted hierarchy in a medium with a vanishing $\Delta V_{\mu \tau}$ (upper panel) and a large $\Delta V_{\mu \tau}$ with 23-mixing in the first octant (central panel) or the second octant (lower panel). The arrows indicate the transitions caused by collective flavor transformations.

the $\bar{\nu}_{e}$ 's leave the star as $\bar{\nu}_{1}$. As a consequence, the survival probability is $P\left(\nu_{e} \rightarrow \nu_{e}\right) \approx \sin ^{2} \Theta_{12}$ and $P\left(\bar{\nu}_{e} \rightarrow \bar{\nu}_{e}\right) \approx$ $\sin ^{2} \Theta_{13}$ or $\cos ^{2} \Theta_{12}$ for large and small $\Theta_{13}$, respectively.

In the presence of neutrino-neutrino interactions, important collective effects take place in the inner SN layers, where the neutrino density is high. We observe in the first two panels of Fig. 2 that collective pair transformations convert the $\nu_{e}$ and $\bar{\nu}_{e}$ fluxes to $\nu_{\tau}^{\prime}$ and $\bar{\nu}_{\tau}^{\prime}$ as indicated by the arrows in the upper panel of Fig. 3. The consequences for the subsequent evolution are dramatic. In the case of $\nu_{e}$ a fraction equal to $\epsilon F_{\bar{\nu}_{e}}$ stays in $\nu_{2}^{\mathrm{m}}$ and evolves as in the absence of neutrino-neutrino interactions, while the rest of $\nu_{e}$ are transformed to $\nu_{3}^{\mathrm{m}}$. As a consequence, the final $\nu_{e}$ flux, normalized to the initial $\bar{\nu}_{e}$ one, is expected to be approximately $\epsilon \sin ^{2} \Theta_{12} \simeq 0.08$, see thick line in the upper left panel in Fig. 2. In the case of antineutrinos the effect of the collective pair conversion is to interchange the eigenstates in which $\bar{\nu}_{e}$ and $\bar{\nu}_{\tau}^{\prime}$ arrive at the H-resonance. Now $\bar{\nu}_{e}$ enters the resonance as $\bar{\nu}_{1}^{\mathrm{m}}$ instead of $\bar{\nu}_{3}^{\mathrm{m}}$. Therefore, for $\sin ^{2} \Theta_{13} \gtrsim 10^{-3}$ the resonance is adiabatic and the $\bar{\nu}_{e}$ 's leave the star as $\bar{\nu}_{1}$, leading to a final normalized flux of approximately $\cos ^{2} \Theta_{12} \simeq 0.68$, see the thick line in the second panel in Fig. 2. Instead, if $\sin ^{2} \Theta_{13} \lesssim 10^{-5}$ again there is a jump of matter eigenstates from $\bar{\nu}_{1}^{\mathrm{m}}$ to $\bar{\nu}_{3}^{\mathrm{m}}$ at the $\mathrm{H}$-resonance. In this case $\bar{\nu}_{e}$ leaves the star as $\bar{\nu}_{3}$, leading to a normalized $\bar{\nu}_{e}$ flux equal to $\sin ^{2} \Theta_{13}$.

The impact of collective effects is easier to understand if we follow the previous literature $[10,12]$ and observe that, in a two-flavor system, the impact of ordinary matter can be transformed away by going into a rotating reference frame for the polarization vectors. Collective conversions proceed in the same way as they would in vacuum, except that the effective mixing angle is reduced. Therefore, assuming an inverted hierarchy (IH) for the atmospheric mass splitting and a normal hierarchy $(\mathrm{NH})$ for the solar splitting, we should consider the level scheme as in the upper left panel of Fig. 4. The mass eigenstates now approximately coincide with the interaction eigenstates because the 23-mixing angle was removed by going to the primed states, and the mixing angles involving $\nu_{e}$ are effectively made small by the presence of matter. Of course, this level scheme does not adiabatically connect to the true vacuum situation.

The initial state consists of $\nu_{e}$ and $\bar{\nu}_{e}$ and thus essentially of $\nu_{1}$ and $\bar{\nu}_{1}$. Collective conversions driven by $\Delta m_{\text {atm }}^{2}$ then transform $\nu_{1} \bar{\nu}_{1}$ pairs to $\nu_{3} \bar{\nu}_{3}$ pairs in the familiar twoflavor way. If both hierarchies are normal, we begin in the lowest-lying state and nothing happens. In the hypothetical case where both hierarchies are inverted (upper right panel in Fig. 4), we begin in the highest state and $\Delta m_{\mathrm{atm}}^{2}$ drives us directly to the lowest state. Finally, if the atmospheric hierarchy is normal and the solar one is inverted (lower right panel in Fig. 4), collective transformations driven by $\Delta m_{\text {sol }}^{2}$ take us to the lowest state.

We have numerically solved the evolution of the threeflavor system with a realistic SN matter profile and found that the results confirm this simple picture. In a two-flavor treatment, the much smaller $\Delta m_{\text {sol }}^{2}$ leads to collective transformations at a much larger radius than $\Delta m_{\mathrm{atm}}^{2}$. In a threeflavor treatment, $\Delta m_{\mathrm{atm}}^{2}$ therefore acts first and takes us directly to the lowest-lying state if the atmospheric hierarchy is inverted. Otherwise only the hypothetical case of 


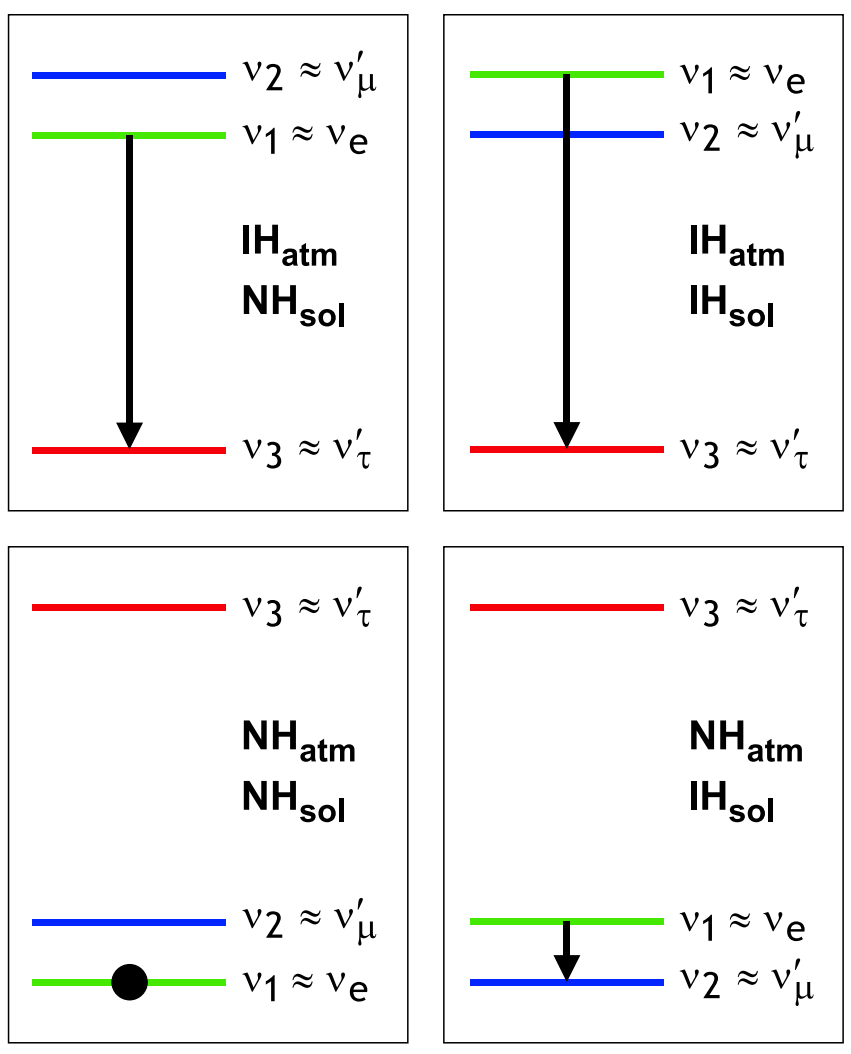

FIG. 4 (color online). Vacuum level diagram for all hypothetical combinations of atmospheric and solar mass hierarchies (normal or inverted). The 12 and 13-mixing angles are assumed to be very small, mimicking the effect of ordinary matter. The effect of collective conversions is indicated by an arrow.

the lower right panel in Fig. 4 is an example where $\Delta m_{\text {sol }}^{2}$ plays any role. We have numerically verified that normal $\Delta m_{\mathrm{atm}}^{2}$ combined with inverted $\Delta m_{\mathrm{sol}}^{2}$ is the only case where $\Delta m_{\mathrm{sol}}^{2}$ drives collective transformations. Since $\Delta m_{\mathrm{sol}}^{2}$ is measured to be normal, the previous two-flavor treatments based on $\Delta m_{\mathrm{atm}}^{2}$ and $\Theta_{13}$ fortuitously capture the full effect.

We conclude that in the limit of a vanishing $\mu \tau$ effect the collective flavor transformations and the subsequent MSW evolution factorize and that the collective effects are correctly treated in a two-flavor picture. Of course, this situation may change if the matter profile is so shallow that the ordinary MSW effects occur in the same region as the collective phenomena [21].

\section{LARGE MU-TAU MATTER EFFECT}

Next we calculate the flavor evolution for the same model, now including a significant $\Delta V_{\mu \tau}$, i.e. we assume a large $\lambda_{0}$. In this case the flavor content of the neutrino and antineutrino fluxes emerging from the SN surface depend on the strength of $\Delta V_{\mu \tau}$ as well as the choice of $\Theta_{23}$, as can be seen in the corresponding panels of Fig. 2. This dependence is best illustrated with the help of the contour plot
Fig. 5 where we show the $\nu_{e}$ and $\bar{\nu}_{e}$ fluxes emerging from the $\mathrm{SN}$, averaged over fast vacuum oscillations.

If $\Delta V_{\mu \tau}$ is so large that the mu-tau effect is strong in the region of collective neutrino oscillations, there are two stable limiting cases, depending on the 23-mixing angle. If the mixing angle is sufficiently nonmaximal and in the first octant, the collective oscillations transform the initially prepared $\nu_{e}$ and $\bar{\nu}_{e}$ fluxes to the propagation eigenstates as indicated by the arrows in the middle panel of Fig. 3, i.e., we observe pair transformations to $\nu_{\tau} \bar{\nu}_{\tau}$.

This behavior is understood if we assume that in the $\mu \tau$ system we can once more go to a rotating frame and now simply imagine that the 23-mixing angle is effectively small by the impact of the $\mu \tau$ matter effect. In this case $\nu_{3} \approx \nu_{\tau}$. Since collective quasivacuum oscillations take us to the lowest-lying state, the $\nu_{3}$ state in the inverted hierarchy, we are effectively taken to $\nu_{\tau} \bar{\nu}_{\tau}$ pairs. Instead, if the 23-mixing angle is in the second octant, $\nu_{\mu}$ and $\nu_{\tau}$ switch roles, explaining that now $\nu_{3} \approx \nu_{\mu}$ and $\bar{\nu}_{3} \approx \bar{\nu}_{\mu}$.

These are only heuristic explanations. We expect that they can be made precise in a true analytic three-flavor treatment of collective neutrino oscillations along the lines of Ref. [22].

For intermediate values of $\Delta V_{\mu \tau}$ and for 23-mixing angles near maximal, the final fluxes depend sensitively on parameters. For intermediate values of $\Delta V_{\mu \tau}$, there are also nontrivial effects for the normal hierarchy. The collective effects do not place the ensemble into propagation eigenstates, preventing a simple interpretation. The sensitive dependence for intermediate $\Delta V_{\mu \tau}$ is also illustrated in Fig. 6 where we show the emerging average $\nu_{e}$ and $\bar{\nu}_{e}$ fluxes as functions of $\lambda_{0}$ for two values of $\Theta_{23}$, one in the first and the other in the second octant. In Fig. 7 we show the same $\nu_{e}$ and $\bar{\nu}_{e}$ fluxes as functions of $\sin ^{2} \Theta_{23}$ for $\lambda_{0}=$ $1.85 \times 10^{9} \mathrm{~km}^{-1}$. One can notice how the fall of $\bar{\rho}_{e e}$ is not exactly centered at $\sin ^{2} \theta_{23}=0.5$ but slightly shifted to smaller values. This is due to second-order corrections to the $\mu \tau$ resonance condition.

This dependence on the $\Theta_{23}$ octant leads to a clear imprint on the final survival probability. Let us first consider the first octant. In the case of $\nu_{e}$ a fraction equal to $\epsilon F_{\bar{\nu}_{e}} \quad$ stays in $\nu_{2}^{\mathrm{m}}$. However the presence of the $\mu \tau$-resonance in the neutrino channel makes the rest of the $\nu_{e}$ to be transformed to $\nu_{1}^{\mathrm{m}}$. Their subsequent evolution would depend on the adiabaticity of the $\mu \tau$-resonance, but it has been shown to be always adiabatic [4]. As a consequence, the final $\nu_{e}$ flux is expected to be approximately $\cos ^{2} \Theta_{12}+\epsilon \sin ^{2} \Theta_{12} \simeq 0.76$, see thick line in the left panel of the second row in Fig. 2. In the case of antineutrinos the situation is completely analogous to the case of vanishing $\Delta V_{\mu \tau}$ so that $P\left(\bar{\nu}_{e} \rightarrow \bar{\nu}_{e}\right) \approx \cos ^{2} \Theta_{12}$ or $\sin ^{2} \Theta_{13}$, depending on the value of $\Theta_{13}$.

If $\Theta_{23}$ belongs to the second octant, then the $\mu \tau$-resonance lies in the antineutrino channel. The crucial point is that now all $\bar{\nu}_{e}$ are transformed to $\bar{\nu}_{\mu}=\bar{\nu}_{2}^{\mathrm{m}}$ before 
IH neutrinos

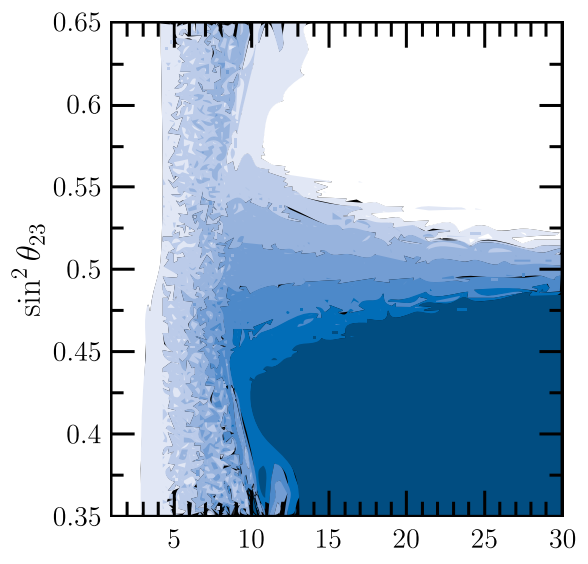

IH antineutrinos

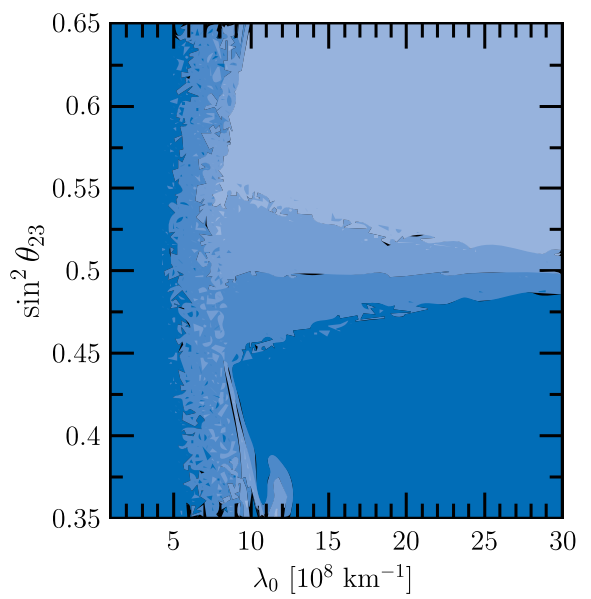

$\mathrm{NH}$ neutrinos

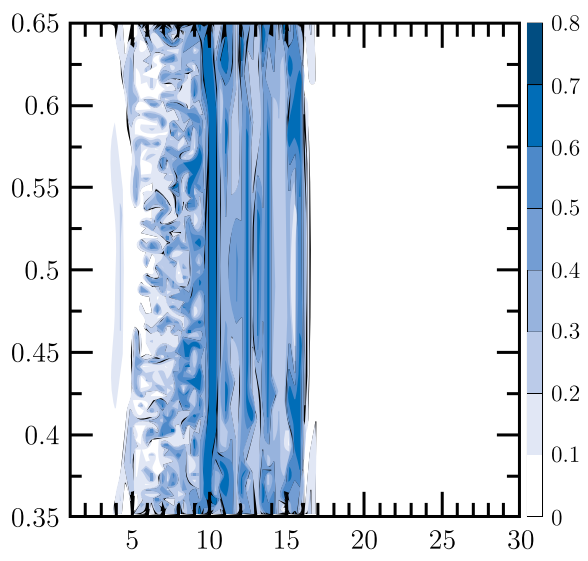

$\mathrm{NH}$ antineutrinos

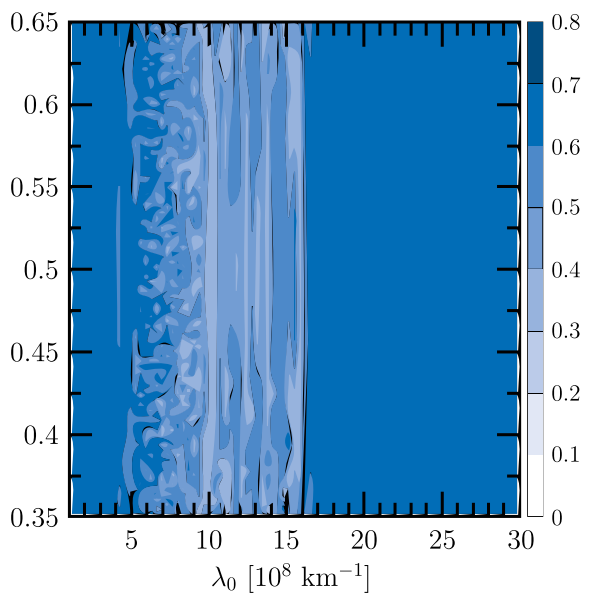

FIG. 5 (color online). Contours in the space of $\sin ^{2} \Theta_{23}$ and $\lambda_{0}$ for the $\nu_{e}$ (top) and $\bar{\nu}_{e}$ (bottom) fluxes emerging from the SN surface for both normal (right) and inverted (left) mass hierarchy. All fluxes are normalized to the initial $\bar{\nu}_{e}$ flux. We show values averaged over fast vacuum oscillations.

reaching the $\mu \tau$-resonance, see the lower panel in Fig. 3. Taking into account that $\bar{\nu}_{2}^{\mathrm{m}}$ does not encounter the $\mathrm{H}$ resonance, the survival probability will be always $P\left(\bar{\nu}_{e} \rightarrow\right.$ $\left.\bar{\nu}_{e}\right) \approx \sin ^{2} \Theta_{12}$, independently of the value of $\Theta_{13}$. On the other hand neutrinos do not feel the $\mu \tau$-resonance and therefore their propagation is the same as in the vanishing $\Delta V_{\mu \tau}$ case.

We present in Table I a summary of the cases discussed so far. One can see the importance of the presence of collective neutrino effects, as well as the dependence on the strength of the mu-tau matter effect.

Another interesting feature concerns the position of $r_{\text {syn }}$ in the presence of a large $\mu \tau$ matter effect. As can be seen comparing the first two rows of Fig. 2, the radius where collective neutrino transformations begin is slightly larger $\left(r_{\mathrm{syn}} \simeq 115 \mathrm{~km}\right)$ when we include a significant $\Delta V_{\mu \tau}$. We have checked that, while for a small $\Delta V_{\mu \tau}$ the position of $r_{\text {syn }}$ is independent of $\Theta_{23}$, for a large $\Delta V_{\mu \tau}$ the onset of bipolar transformations is delayed for nonzero values of $\Theta_{23}$. This effect is largest for maximal mixing $\left(\Theta_{23}=\right.$ $\pi / 4)$ and symmetric relative to $\Theta_{23}=\pi / 4$. A full understanding of this variation presumably requires an analytic three-flavor treatment in the spirit of Ref. [22].

TABLE I. Summary of the approximate values of the $\bar{\nu}_{e}$ survival probability for an inverted hierarchy, including or not collective effects. Here a small (large) mixing angle $\Theta_{13}$ stands for $\sin ^{2} \Theta_{13} \lesssim 10^{-5}\left(\sin ^{2} \Theta_{13} \gtrsim 10^{-3}\right)$, while a small (large) $\Delta V_{\mu \tau}$ represents $r_{\mu \tau}$ being smaller (larger) than $r_{\text {syn }}$.

\begin{tabular}{|c|c|c|c|c|c|}
\hline Collective effect & $\Delta V_{\mu \tau}$ & $\Theta_{23}$ & $\Theta_{13}$ & $\bar{\nu}_{e}$ leaves as & $P\left(\bar{\nu}_{e} \rightarrow \bar{\nu}_{e}\right)$ \\
\hline no & any & any & small & $\bar{\nu}_{1}$ & $\cos ^{2} \Theta_{12}$ \\
\hline no & any & any & large & $\bar{\nu}_{3}$ & $\sin ^{2} \Theta_{13}$ \\
\hline yes & small & any & small & $\bar{\nu}_{3}$ & $\sin ^{2} \Theta_{13}$ \\
\hline yes & small & any & large & $\bar{\nu}_{1}$ & $\cos ^{2} \Theta_{12}$ \\
\hline yes & large & $<\pi / 4$ & small & $\bar{\nu}_{3}$ & $\sin ^{2} \Theta_{13}$ \\
\hline yes & large & $<\pi / 4$ & large & $\bar{\nu}_{1}$ & $\cos ^{2} \Theta_{12}$ \\
\hline yes & large & $>\pi / 4$ & any & $\bar{\nu}_{2}$ & $\sin ^{2} \Theta_{12}$ \\
\hline
\end{tabular}



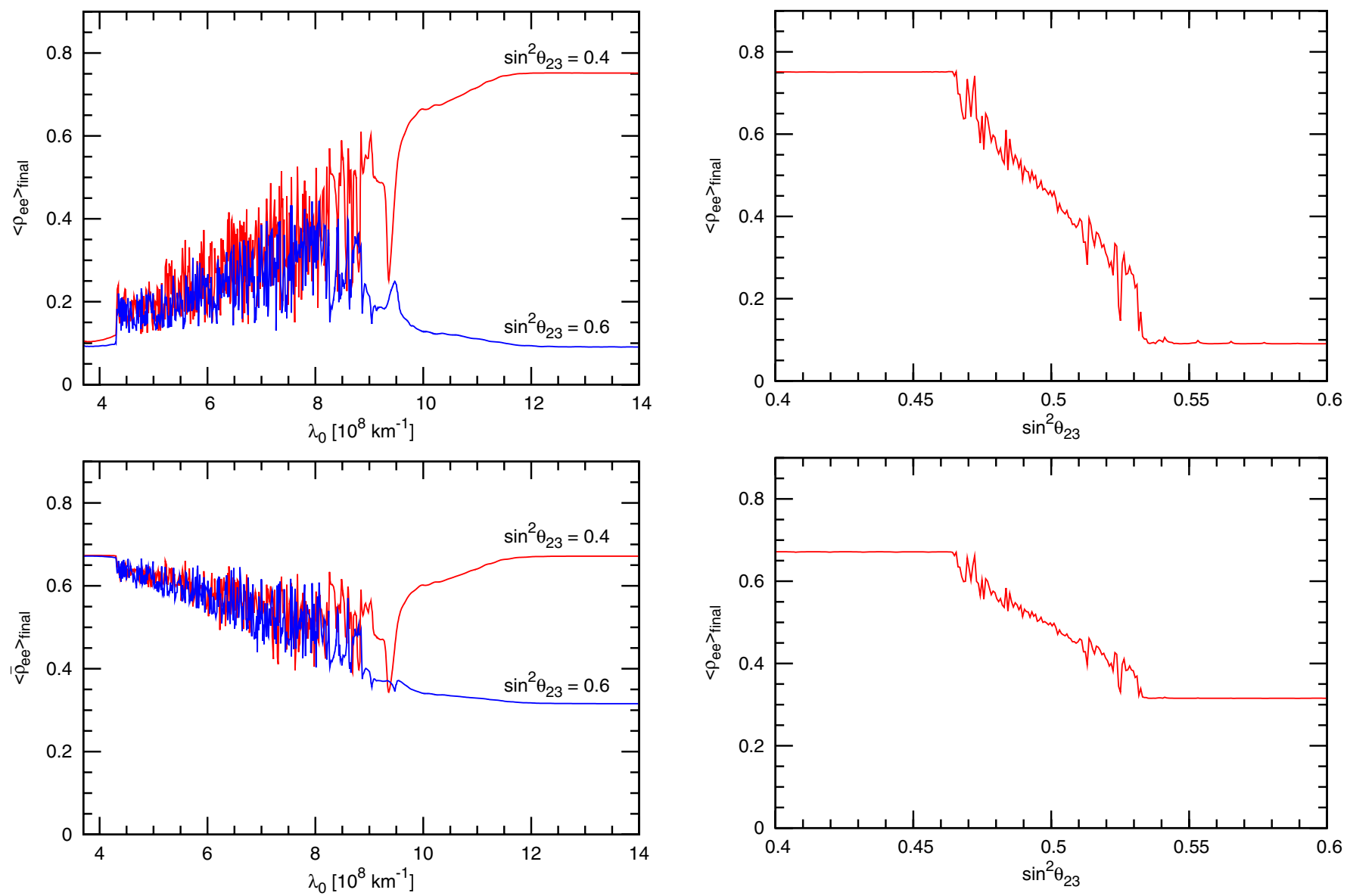

FIG. 6 (color online). Fluxes of $\nu_{e}$ (top) and $\bar{\nu}_{e}$ (bottom), normalized to the initial $\bar{\nu}_{e}$ flux, emerging from the $\mathrm{SN}$ as a function of $\lambda_{0}$ for a 23-mixing angle in the first (red line) or second (blue line) octant. These curves represent cuts through the inverted hierarchy contour plots of Fig. 5 at the indicated values of $\sin ^{2} \Theta_{23}$.

\section{CONCLUSIONS}

At the relatively low energies relevant for $\mathrm{SN}$ neutrinos, charged mu and tau leptons cannot be produced so that muand tau-flavored neutrinos are not distinguishable in the SN or in detectors. (In the inner core of a SN the temperatures may be high enough to produce a significant thermal muon density, but this would not affect the emission from the neutrino sphere.) The impact of the small second-order difference between the $\nu_{\mu}$ and $\nu_{\tau}$ refractive index does not produce observable effects as long as one only considers the traditional MSW flavor conversion [4].

The picture changes if one includes the unavoidable effect of collective neutrino transformations in the region above the neutrino sphere. If the matter density is large enough that $\Delta V_{\mu \tau}$ is comparable to or larger than $\Delta m_{\mathrm{atm}}^{2} / 2 E$, the survival probability of $\nu_{e}$ and $\bar{\nu}_{e}$ can be completely modified and depends sensitively on the mixing angle $\Theta_{23}$. In the future one should also include nonmonochromatic energy spectra, leading to spectral split

FIG. 7 (color online). Fluxes of $\nu_{e}$ (top) and $\bar{\nu}_{e}$ (bottom), normalized to the initial $\bar{\nu}_{e}$ flux, emerging from the $\mathrm{SN}$ as a function of $\sin ^{2} \Theta_{23}$ for $\lambda_{0}=1.85 \times 10^{9} \mathrm{~km}^{-1}$. These curves represent cuts through the inverted hierarchy contour plots of Fig. 5 at the indicated value of $\lambda_{0}$.

phenomena that could be more complicated than the previously studied two-flavor cases. One should also explore the impact of realistic angular distributions and of a nonzero Dirac phase in the neutrino mixing matrix [27].

Lower-mass progenitors may collapse with a $\mathrm{O}-\mathrm{Ne}-\mathrm{Mg}$ core and, on the computer, explode easily because there is very little mass in the envelope [28]. Even at core bounce and immediately afterward, the density profile is so shallow that the ordinary $\mathrm{H}$ - and L-resonances may occur within the collective neutrino region [21]. In this case the effects discussed here are irrelevant because the mu-tau matter effect is negligible. Probably our effects are also negligible during the cooling phase of an iron-core SN. However, flavor oscillation effects are probably largest during the accretion phase of an iron-core $\mathrm{SN}$ where the flavor dependence of the spectra and fluxes is more pronounced than during the cooling phase [29].

When it is important, the mu-tau matter effect adds one more layer of complication to the already vexed problem of collective SN neutrino oscillations. It was previously recognized that "ordinary" collective oscillations are almost 
completely insensitive to the smallness of $\Theta_{13}$ as long as it is not exactly zero. Here we have found the opposite for the large mixing angle $\Theta_{23}$ that is often assumed to be maximal. Even small deviations from maximal 23-mixing can imprint themselves in the collective oscillation effect. Both results are counter-intuitive and opposite to ordinary flavor oscillations.

\section{ACKNOWLEDGMENTS}

We thank E. Akhmedov for an illuminating correspondence, A. Mirizzi for comments on the manuscript, and B. Dasgupta and A. Dighe for helpful comments and for making their manuscript [22] available before completion. This work was partly supported by the Deutsche Forschungsgemeinschaft (Grant No. TR-27 "Neutrinos and Beyond"), by the Cluster of Excellence "Origin and Structure of the Universe" (Garching and Munich), by the European Union (Contracts No. RII3-CT-2004-506222 and No. MRTN-CT-2004-503369), and by the Spanish Grants No. FPA2005-01269 (MEC) and No. ACOMP07-270 (Generalitat Valenciana). A. E. was supported by an FPU grant from the Spanish Government. S.P. and R. T. were supported by MEC contracts (Ramón y Cajal and Juan de la Cierva, respectively).
[1] L. Wolfenstein, Phys. Rev. D 17, 2369 (1978).

[2] F. J. Botella, C. S. Lim, and W. J. Marciano, Phys. Rev. D 35, 896 (1987).

[3] E. Roulet, Phys. Lett. B 356, 264 (1995).

[4] E. Kh. Akhmedov, C. Lunardini, and A. Yu. Smirnov, Nucl. Phys. B643, 339 (2002).

[5] A. Arcones, H.T. Janka, and L. Scheck, Astron. Astrophys. 467, 1227 (2007).

[6] A. S. Dighe and A. Yu. Smirnov, Phys. Rev. D 62, 033007 (2000).

[7] S. Pastor and G. G. Raffelt, Phys. Rev. Lett. 89, 191101 (2002).

[8] R.F. Sawyer, arXiv:hep-ph/0408265.

[9] R. F. Sawyer, Phys. Rev. D 72, 045003 (2005).

[10] H. Duan, G. M. Fuller, and Y.Z. Qian, Phys. Rev. D 74, 123004 (2006).

[11] H. Duan, G. M. Fuller, J. Carlson, and Y.Z. Qian, Phys. Rev. D 74, 105014 (2006).

[12] S. Hannestad, G. G. Raffelt, G. Sigl, and Y. Y. Y. Wong, Phys. Rev. D 74, 105010 (2006).

[13] G. G. Raffelt and G. Sigl, Phys. Rev. D 75, 083002 (2007).

[14] H. Duan, G. M. Fuller, J. Carlson, and Y.Z. Qian, Phys. Rev. D 75, 125005 (2007).

[15] G. G. Raffelt and A. Yu. Smirnov, Phys. Rev. D 76, 081301 (2007).
[16] G. G. Raffelt and A. Yu. Smirnov, Phys. Rev. D 76, 125008 (2007).

[17] A. Esteban-Pretel, S. Pastor, R. Tomàs, G. G. Raffelt, and G. Sigl, Phys. Rev. D 76, 125018 (2007).

[18] H. Duan, G. M. Fuller, and Y.Z. Qian, Phys. Rev. D 76, 085013 (2007).

[19] H. Duan, G. M. Fuller, J. Carlson, and Y.Z. Qian, Phys. Rev. Lett. 99, 241802 (2007).

[20] G. L. Fogli, E. Lisi, A. Marrone, and A. Mirizzi, J. Cosmol. Astropart. Phys. 12 (2007) 010.

[21] H. Duan, G. M. Fuller, J. Carlson, and Y.Z. Qian, Phys. Rev. Lett. 100, 021101 (2008).

[22] B. Dasgupta and A. Dighe, arXiv:0712.3798.

[23] G. Sigl and G. G. Raffelt, Nucl. Phys. B406, 423 (1993).

[24] M. Maltoni, T. Schwetz, M. A. Tórtola, and J. W. F. Valle, New J. Phys. 6, 122 (2004).

[25] G. L. Fogli, E. Lisi, A. Marrone, and A. Palazzo, Prog. Part. Nucl. Phys. 57, 742 (2006).

[26] M. C. González-García and M. Maltoni, arXiv:0704.1800 [Phys. Rep. (to be published].

[27] A. B. Balantekin, J. Gava, and C. Volpe, arXiv:0710.3112.

[28] F. S. Kitaura, H.-T. Janka, and W. Hillebrandt, Astron. Astrophys. 450, 345 (2006).

[29] M. T. Keil, G. G. Raffelt, and H. T. Janka, Astrophys. J. 590, 971 (2003). 